\title{
LA RELACIÓN DE COMPRAVENTA EN LA PROSTITUCIÓN
}

\author{
PALOMA MARTÍN MARTÍN ${ }^{1}$ \\ CARMEN MENESES FALCÓN ${ }^{2}$
}

Fecha de recepción: agosto de 2020

Fecha de aceptación y versión definitiva: septiembre de 2020

\begin{abstract}
RESUMEN: Este trabajo tiene la finalidad de reflexionar sobre los hombres que pagan servicios sexuales en un momento que se reclama su criminalización desde sectores feministas. La reflexión parte de los estudios previos realizados tanto nacionales como internacionales. Se pone de relieve las características, motivaciones, perfiles y demandas de estos hombres. Se señalan los riesgos que la relación de compraventa puede generar especialmente para las mujeres. Se revisan los modelos de intervención llevados a cabo con estos hombres y se proponen intervenciones socioeducativas y sanitarias por ser más efectivas tanto en la disminución de riesgo como en la reducción de la demanda.
\end{abstract}

PALABRAS CLAVE: prostitución; clientes; intervención social; sexualidad y trata sexual.

\section{The relationship between sale and purchase in prostitution}

ABSTRACT: This work aims to reflect on men who pay for sexual services at a time when there are calls for their criminalization from feminist sectors. The reflection is based on previous studies, both national and international. It highlights the characteristics, motivations, profiles and demands of these men. The risks that the buying and selling relationship can generate especially for women are pointed out. The models of intervention carried out with these men are reviewed and socio-educational and health interventions are proposed as they are more effective in both risk reduction and demand reduction.

KEY WORDS: prostitution; clients; social intervention; sexuality; sexual trafficking.

1 Universitat de les Illes Balears, Facultad de Filosofía y Letras. Correo electrónico: paloma.martin@uib.es.

2 Universidad Pontificia Comillas, Facultad Ciencias Humanas y Sociales. Correo electrónico: cmeneses@comillas.edu. 


\section{INTRODUCCIÓN}

Este trabajo tiene la finalidad de reflexionar sobre los hombres que pagan servicios sexuales en un momento de controversia y reclamo de su criminalización desde el feminismo abolicionista. La reflexión se basa en estudios previos realizados tanto nacionales como internacionales además de identificar el trabajo que desarrollan algunas organizaciones de nuestro país.

La mayoría de los estudios sobre prostitución o trabajo sexual ${ }^{3}$ se centran en las mujeres con enfoques muy diferente (criminológico, de salud, desviación, moral, etc.) pero escasamente la mirada se dirige a la otra parte que interviene en la relación prostitucional, los compradores (Meneses, 2010a). La compraventa de servicios sexuales es un fenómeno que puede ser analizado desde muchos enfoques, entre los que encontramos el de género (De Miguel y Torrado, 2104). Hay un hecho que se repite en las encuestas realizadas, tanto nacionales como internacionales, y es que la demanda de servicios sexuales es primordialmente masculina mientras que la oferta es mayoritariamente femenina (CIS, 2009). Y aunque también personas transexuales o varones venden servicios sexuales, encontramos que sus principales clientes también suelen ser hombres (Hor, Detels, Heng y Mun., 2005; Mulieri et al., 2010).

No podemos abordar el análisis sin tener en cuenta las desiguales oportunidades de mujeres y hombres en nuestra sociedad, así como las diferencias de clase y de origen que contribuyen a que se ocupen distintos lugares en torno al sexo. Si tenemos en cuenta las opciones y oportunidades que las mujeres disponen en las sociedades actuales, y a pesar de que se ha avanzado mucho en participación y visibilidad, las desigualdades económicas, de poder, de acceso a los recursos y de responsabilidades sigue siendo elementos pendientes en la agenda para alcanzar la igualdad. A pesar de las leyes igualitarias continúan existiendo diferentes mecanismos que perpetúan tales desigualdades (De Miguel, 2014), de manera que las opciones de muchas mujeres son limitadas e insuficientes. En ocasiones la venta de sexo se puede convertir en una opción de adquisición de recursos económicos importante para algunas mujeres (Neira, 2012), sobre todo para aquellos sectores de la sociedad más vulnerables, con mayor dificultad de acceso al mercado laboral, o con escasa capacitación y habilidades laborales. Y es que «la prostitución no es un fenómeno aislado, sino un síntoma visible de la situación general

3 Se utilizan ambos conceptos o indistintamente debido a que ambos están presentes en la literatura revisada. A pesar de ello, somos conscientes de las diferencias existentes entre ambos, del posicionamiento que cada uno representa y la lejanía ideológica entre uno y otro. 
de la mujer dentro de una sociedad, o de la situación de ciertos colectivos de mujeres dentro de una sociedad global»(Juliano, 2002, p. 12).

Por otro lado, el sexo es algo que los hombres desean y hacía lo que muestran un mayor interés, siendo proporcionado por las mujeres y suponiéndoles una fuente importante de recursos económicos y de bienes (Baumeister y Voh, 2004). Y aunque las mujeres pueden compran estos servicios en los que se producen aspectos similares en este tipo de intercambios de sexo por dinero, pues también existen relaciones desiguales (Berg, Molin y Nanavati, 2020), la realidad y las cifras expresan que se realiza de una manera minoritaria.

Teniendo en cuenta estas diferencias, se hace imprescindible analizar e intervenir en el fenómeno incorporando la perspectiva de género, para entender porque se producen estas diferencias e intentar disminuir las desigualdades y discriminaciones que estos intercambios generan y que casi siempre son desfavorables para las mujeres. Incorporar la perspectiva de género no supone estudiar únicamente la realidad de las mujeres, sino analizar las relaciones existentes entre las personas que interaccionan en este intercambio. Esto implica estudiar el comportamiento de los hombres, en este caso, en relación con el consumo de sexo, que no es paralelo al feminismo, sino que forma parte de la estrategia feminista para comprender el «orden del género» (Connell, 2012, p. 12). Del mismo modo, la intervención con perspectiva de género, no supone centrarse únicamente en las mujeres para modificar su situación aisladamente, sino realizar intervenciones que puedan generar cambios importantes en las y los actores sociales, dónde ocupan un lugar relevante los hombres que compran estos servicios.

Esta diferenciación del género nos hace reflexionar sobre diferentes factores. No podemos entender la compraventa de sexo sin analizar su vinculación con la sexualidad, el placer, la percepción, sentimiento y experiencia de los hombres y de las mujeres en las relaciones afectivo-sexuales. Sin olvidar que la llamada "conducta sexual» es fruto de un proceso de socialización (Millett, 2018), de manera que, está atravesada por variables socioculturales y factores estructurales en donde las relaciones de poder y jerárquicas son elementos fundamentales en el análisis.

En una sociedad patriarcal, la sexualidad se entiende y se establece en base a los valores de los hombres (Cobo, 2016). La sexualidad masculina es el eje central en el que las instituciones sociales y culturales se desarrollan y perpetúan, y a su vez, instituciones como la prostitución y la pornografía pueden suponer un espacio de socialización masculina que permite reafirmar una identidad masculina (Alario, 2018), que resulta imprescindible para el mantenimiento de la estructura patriarcal. Por ello, ha sido seriamente 
considerada la vinculación entre sexualidad y prostitución, pues la identidad masculina se realiza, negocia y reproduce en el contexto de la prostitución (Huysamen, 2016).

Sin embargo, la sexualidad masculina es mucho más amplia, como lo ha puesto de relieve Connell en sus distintos trabajos (Connell, 2008, y 2012). Como no debemos hacerlo con las mujeres, tampoco podemos generalizar la situación de los hombres. La variedad de circunstancias, experiencias y percepciones no son únicas en todo ellos, sino que, frente a una masculinidad prevalente o hegemónica, emergen otras sexualidades menos visibles y valoradas que llevan a entender a los hombres y su sexualidad de manera plural. Investigaciones previas nos muestran como dentro de los servicios sexuales encontramos a varones que responden a otros y nuevos modelos de masculinidad (Kong, 2015; Joseph y Black, 2012). El análisis que se debe acometer en este tema es entre los hombres y entre éstos y las mujeres.

En un momento de profundo debate sobre la prostitución y el trabajo sexual se ha volcado la mirada hacia los hombres que pagan por estos servicios como responsables de la existencia de la trata de seres humanos con fines de explotación sexual. Dos posiciones diferente hallamos en los debates y discursos en torno a los clientes. Por un lado, el abolicionismo conceptualiza al cliente como responsables de la existencia de la trata con su consumo, pues si no hubiera demanda no habría oferta. Además, de considerar la demanda de servicios sexuales como una clara representación de violencia de género porque se plantea este intercambio en relaciones desiguales económicas y sociales (De Miguel, 2014; Cobo, 2017). Por otro, el movimiento pro-sex ha descrito la demanda de servicios sexuales masculina como un recurrente universal que se contrapone con el escaso interés que las mujeres muestran por las relaciones sexuales en términos de frecuencia en ciertas etapas de su vida, tal y como lo señala Hakim (2010) en la revisión de encuestas sexuales nacionales e internacionales. Además de conceptualizarlo como una actividad económica más que en el capitalismo actual adquiere características diferentes a la antigüedad, donde existe una mercantilización de los servicios personales, entre los que se encuentran los servicios sexuales (Giddens,1992).

Por otro lado, una serie de cambios culturales y sociales han modificado el concepto de sexualidad y del sexo en las sociedades contemporáneas, influyendo una multitud de situaciones que hacen forjarse una cultura del sexo diferente a las sociedades pasadas (Sanders, 2008).

Partiendo de la premisa de que no existe una única masculinidad, y de que las diferentes masculinidades son modificables y cambiantes (Connell, 2012), se nos presenta como necesario colocar en el centro de la investigación sobre sexo de pago a los varones que pagan por servicios sexuales. Analizar 
su comportamiento en contextos de compraventa de sexo y generar líneas de intervención sociosanitarias y educativas que permitan un cambio hacia una sociedad con mayor equidad entre los sexos y géneros.

Este trabajo reflexiona sobre un tipo de masculinidad y conducta masculina: la compra de servicios sexuales en hombres heterosexuales. Se pretende revisar algunos de los factores y circunstancias que se asocian con esta conducta y sugerir líneas de intervención que pueden conllevar un cambio en las relaciones desiguales entre hombres y mujeres, así como una reducción de algunos de los riesgos asociados a la venta de servicios sexuales.

\section{LOS VARONES QUE PAGAN POR SERVICIOS SEXUALES}

Diferenciar a los hombres que pagan servicios sexuales de aquellos que no lo hacen resulta complejo, porque es un objetivo que no se ha considerado en profundidad, y aquellos que lo han abordado no han encontrado diferencias significativas importantes (Meneses, Rúa y Uroz, 2018a). Sin embargo, hay algunos datos que poseemos sobre este grupo social en estudios realizados en España.

En primer lugar, las encuestas realizadas en INE indicaban una conducta que se producía alguna vez en la vida en el 25,4\% de los varones entre de 18 a 49 año y el 5,7\% en los 12 últimos meses (Belza et al., 2008). En un estudio más reciente, con una muestra aleatoria nacional, se señaló que el 20,3\% habían pagado alguna vez y el 15\% de los hombres entre 18 y 70 años en el último año (Meneses et al., 2018a). Como es lógico la prevalencia de vida es mayor frente a la prevalencia anual, siendo más estable esta últimas. Que muchos hombres hayan pagado alguna vez por sexo, especialmente en los procesos de ritos de paso o de manifestación de la virilidad y heterosexualidad entre iguales, no significa que desde entonces continúe con esa conducta. En ese sentido la prevalencia de los últimos doce meses indica una mayor cercanía y en estos casos son siempre menores. Por tanto, las encuestas representativas o aleatorias nos muestran que la mayoría de los españoles, $u$ hombres residentes en España, no muestran mayoritariamente esta conducta de pago por servicios sexuales.

En segundo lugar, respecto a las características sociodemográficas los hombres que pagan por sexo no muestran un perfil diferente (Baringo Ezquerra y López Insausti, 2007; Gómez-Suárez y Verdugo-Matés, 2016; Arjona, Checa y Majuelos, 2019). Se podría mencionar que en la muestra de Meneses et al. (2018a) señalaron una serie de diferencias significativas: los 
que pagaban servicios sexuales mostraron menor nivel educativo, menor proporción de pareja estable, menor nivel de satisfacción vital y sexual (aunque en ambos casos era alta), y una edad media mayor (47 años) que los que no pagaron por sexo. Hasta la fecha no existen datos que nos hagan señalar que este grupo social masculino tenga unas características diferenciadas.

En tercer lugar, se ha investigado las motivaciones que están detrás del pago de servicios sexuales. En el estudio realizado en el País Vasco se señaló que obtener sexo con distintas mujeres, tener sexo sin compromiso, obtener prácticas sexuales distintas, vida sexual insatisfactoria o la imposibilidad de mantener relaciones de otro modo eran razones más frecuentes (Askabide, 2008). En el estudio de Galicia se proponía que las motivaciones eran fisiológicas (necesidad sexual), socioculturales (socializarse, amigos, etc.), lúdicas (divertirse, fantasías sexuales) y carencias afectivas (Gómez Suárez, Día-ALECRIN y Pérez Freire, 2009). En la investigación realizada en Madrid, se apuntaba a una serie de factores motivacionales: conseguir compañía, la necesidad de obtener sexo y placer, una manera de distracción, la atracción hacia el riesgo que supone mantener relaciones sexuales con desconocidos, la dominación hacia las mujeres o la rapidez en conseguir relaciones sexuales evitando el flirteo (Meneses, 2010a). Por último, en el trabajo realizado en Almería, se indicaban algunas razones que se hallaban detrás de esta conducta: la búsqueda y satisfacción sexual cuando existía ausencia de relaciones sexuales y la ausencia de pareja que proporcione esas relaciones, también como una forma de ocio, o para cubrir aspectos comunicativos o terapéuticos, es decir, poder desahogarse de situaciones vitales estresantes (Majuelos et al., 2019). Por tanto, de todos ellos podemos decir que se unen a la mera petición de una práctica sexual otras razones que a veces le acompañan o que adquieren su prioridad. En algunos varones el conseguir compañía femenina que le escuche y con quien pasar un rato de ocio puede ser más importante que el sexo, mientras que otros solo desean diferentes prácticas sexuales con una diversidad de mujeres.

En cuarto lugar, se han generado distintas tipologías de clientes: a) se han diferenciado entre "personalizadores» $\mathrm{y}$ «objetualizadores» distinguiendo principalmente cual es la relación que tienen con la persona que ofrece servicios sexuales (Solana, 2003); b) se han distinguido entre misóginos, consumidores, amigos y críticos dependiendo de cuál fuera su mirada hacia el comercio sexual, siendo el último el cliente que a pesar de haber pagado por servicios reconoce no estar convencido de ello (Gómez Suárez, Pérez Freire y Verdugo Matés, 2015); c) se han diferenciado en función de lo que buscan en la relación comercial distinguiéndose entre, Funners, que buscan ocio y diversión, los Thingers sexo sin compromiso, los Looker for couple alguien 
cercano a una pareja, Risker comportamientos de riesgo y Personalizers que desean sexo con intimidad y compañía (Meneses et al., 2018a). Esta diversidad nos permite pensar en que, a pesar de colocar a todos los hombres en un mismo grupo, existe una gran heterogeneidad entre ellos, distanciando muchos sus perfiles.

En quinto lugar, y a pesar de que no siempre los hombres pagan por sexo, las prácticas más demandadas identificadas han sido: sexo oral $(68,1 \%)$, sexo vaginal $(59,6 \%)$, masturbación $(33,3 \%)$, penetración anal insertiva $(22,5 \%)$, penetración anal receptiva $(21,7 \%)$, trío-dúplex $(16,7 \%)$, uso de juguete sexual $(12,3 \%)$, lluvia dorada $(6,5 \%)$, sexo sado $(5,8 \%)$, sexo-dominación $(4,3 \%)$, sexo con puño $(2,9 \%)$, sexo con violencia $(2,9 \%)$ y sexo-sumisión $(2,2 \%)$ (Meneses y Rua Vieites, 2011). Algunas de estas prácticas pueden suponer riesgos en sí, y otras lo son en el caso de realizarse sin protección. El sexo desprotegido, aunque no se produce por la totalidad de los clientes ni de los servicios (Belza et al., 2008), es una petición frecuente por parte de muchos de los hombres, llegando a ser lograda en muchas ocasiones (Meneses y Rua Vieites, 2011). Este tipo de situaciones sin duda pone en peligro no solo la salud del demandante sino especialmente de la persona que ofrece los servicios sexuales. La presión económica que se produce para obtenerlo es un reclamo importante para muchas mujeres (Meneses, 2007a). Por último, el consumo de alcohol o cocaína, entre otras sustancias psicoactivas, han sido referenciados en diversos trabajos (Meneses, 2007a y 2010b). Estos aspectos relacionados con el riesgo los abordaremos en el punto siguiente.

\section{EL CLIENTE COMO RIESGO}

La compraventa de servicios sexuales conlleva una serie de riesgos para los actores implicados, sin embargo, no puede valorarse de la misma manera en todos ellos ni los riesgos son iguales. Las relaciones de poder y asimétricas desarrollan distinta percepción, valoración y gestión de los riesgos (Meneses, 2007b). Hay riesgos que son aceptados y otros por el contrario no, algunos son controlables y otros no. ¿Son los hombres que pagan por sexo un agente de riesgo para las personas que ejercen la prostitución, más en concreto para las mujeres? Diversos trabajos han abordado esta cuestión y han señalado que los riesgos que las mujeres se encuentran en el desarrollo de los servicios sexuales son: las situaciones de violencia (Barnard et al., 2000), los riesgos asociados a la salud (Cusick, 1998; Belza et al., 2008) y entre ellos los asociados con el consumo de drogas (McKeganey y Barnad, 1996; Meneses, 2007a 
y 2010), la utilización de menores o la colaboración con el delito de trata con fines de explotación sexual (Castaño Reyero et al., 2015). Entraremos en cada uno de ellos.

Situaciones de violencia. Las personas que prestan servicios sexuales están expuestas a situaciones de violencia por parte de distintos agentes (la policía, los proxenetas o tratantes, grupos violentos, etc.) pero una de las principales fuentes es el cliente. Si bien es cierto que no parece que sea excesivamente frecuente la presencia de clientes violentos (Solana, 2003; Meneses et al., 2018a), la posibilidad de tener que gestionar situaciones de posible violencia pueden acontecer en distintas circunstancias. La relación que se establece con los hombres es asimétrica y puede provocar violencia. La subordinación de quien ofrece placer a cambio de dinero frente a quién paga por adquirir ese placer desencadenaría también cierto maltrato. En el estudio de Askabide en Vizcaya, de las 373 personas entrevistadas que ejercían la prostitución, 77 reconocieron haber recibido insultos y 13 agresiones físicas (Askabide, 2008). El cliente es capaz de transgredir los acuerdos previos pudiendo llevar a cabo actos agresivos para la consecución de sus objetivos (Moreira y Monteiro, 2012). En la intimidad de las relaciones afectivos-sexuales se puede desarrollar agresiones o situaciones de maltrato. También se ha planteado que los contextos donde se desarrolla la actividad presentan distintos factores de riesgo para sufrir la violencia. En este sentido diversos estudios han mostrado como la prostitución de calle tiene mayor probabilidad de acontecer situaciones de violencia que en los contextos cerrados y con medidas de seguridad (Sanders, 2004; Raphael y Shapiro, 2004). Los motivos expresados en las diversas investigaciones que están detrás de esa violencia son diversos. Algunos como hemos apuntado son intrínsecos a la relación, pues para un sector de los hombres la dominación y la superioridad hacia la persona que le presta los servicios puede llevarle a ejercer situaciones de violencia. Pero en otras ocasiones pueden estar relacionadas con una inadecuada negociación de los servicios sexuales, un abuso de sustancias psicoactivas y la insatisfacción de los servicios ofertados.

Riesgos para la salud. Tenemos que destacar los riesgos que tienen relación directa con el sexo, especialmente con el sexo sin protección. El posible contagio de numerosas infecciones de transmisión sexual, e incluso algunas que pueden transmitirse con la utilización del preservativo, como puede ser el VPH (virus de papiloma humano) son un hecho que deben enfrentar las personas que prestan servicios sexuales. En zonas como África subsahariana en 1993, la infección entre clientes se identificó entre el 8,4\% y el 56\% de los hombres que consumían sexo (Alary et al., 2003). A pesar de que es difícil imaginar que exista desconocimiento sobre las medidas de protección, se 
describe que existen bajos niveles de protección debidos a una conciencia insuficiente sobre el riesgo (Girchenko et al., 2015).

En el propio discurso de las personas que venden sexo se aprecia la complicada negociación con algunos de los varones compradores y las consecuencias de ese incumplimiento en la negociación desembocan en desacuerdos que son acompañados de agresiones. Entre estas discrepancias, en el estudio de Vizcaya se recogió que más del 50\% de las personas que ofrecían sexo recibió la demanda de no utilizar preservativos por parte de sus clientes (Askabide, 2008, p. 42). Sobre las razones para no usar protección se ha mencionado una "visión individualista del riesgo» provocando que la persona que compra sexo no se percibe a sí misma como agente de riesgo (Pardo Herrero, Meroño Salvador y Fundació Àmbit Prevenció, 2015). Otros factores que pueden influir son el aislamiento social, los riesgos relacionados con el lugar, las relaciones sociales y los roles económicos (Goldenberg et al., 2010). Por tanto, la reducción de placer y la escasa percepción de riesgo serían ejes centrales en la no protección (Yang, Yang, Latkin, Luan y Nelson, 2016). La desprotección se justifica con el lugar dominante que ocupa el placer sexual masculino, haciendo que el preservativo sea un elemento no solo que resta ese placer, sino que implica la pérdida de tiempo en colocárselo (Simonetto, 2018). Todo ello provoca que los clientes tengan riesgo potencial en la propagación de enfermedades (Gomes Do Espirito Santo y Etheredge, 2005), tanto hacia las personas que venden sexo como a la población en general (Fleming et al., 2017). A pesar de ello, los varones no suelen participar en pruebas diagnósticas, por lo que se deben replantear intervenciones directamente en zonas donde se encuentra la venta de servicios sexuales (Diserens et al., 2010).

Algunos trabajos han relacionado el consumo de drogas con la compra de servicios sexuales pues son considerados para muchos clientes un espacio de ocio en el que se alternan otros tipos de consumos, que también suponen una fuente de placer (Meneses, 2020). El uso de alcohol y cocaína adquieren una prevalencia importante en algunos de los contextos de servicios sexuales. Se ha señalado que existe una relación entre el consumo excesivo de alcohol y cocaína con la realización de prácticas sexuales desprotegidas (Meneses, 2007a) o con conductas violentas (Majuelos Martínez, 2014). Pero especialmente pueden provocar en las personas que realizan estos servicios un abuso o dependencia a las drogas, pues la petición de consumo suele provenir del cliente.

Colaboración con el delito de la trata y utilización de menores. Por último, se ha señalado que algunos clientes colaboran con los delitos de la trata de seres humanos con fines de explotación sexual y con solicitar los servicios a mujeres menores de edad. No existen estudios al respecto suficientes como 
para plantear su extensión y caracterizar a este tipo de cliente o varón que paga por servicios sexuales transgrediendo la ley. En el estudio de Meneses, Uroz y Rúa (2018b) se ponía de manifiesto que la mayoría de los hombres entrevistados percibían que los servicios sexuales obtenidos eran realizados libremente, sin estar coaccionadas las mujeres con las que alternaron. Solo dos casos, de las diecisiete entrevistas a hombres que pagaron por servicios sexuales, reconocieron detectar menores o mujeres en situación de coacción en el lugar que obtuvieron servicios sexuales. Que un sector pueda priorizar su satisfacción sexual por encima de las condiciones de las mujeres que le prestan los servicios sexuales no significa que sea el causante directo de las situaciones de trata. Esta visión resulta muy reduccionista ya que detrás de la trata con fines de explotación sexual existen además factores estructurales de mayor envergadura que la demanda de servicios sexuales (Weitzer, 2012).

\section{INTERVENCIÓN CON CLIENTES EN CONTEXTOS DE PROSTITUCIÓN}

Las intervenciones llevadas a cabo en relación con la compraventa de sexo habitualmente han estado centradas principalmente en la persona que vende sexo, en la atención sociosanitaria y en la búsqueda de alternativas para el abandono de la prostitución. La intervención desde el trabajo social con personas que venden sexo se remonta a los inicios de la profesión, pero sin ser el varón que paga por sexo objeto de estas intervenciones (Wahab y Sloan, 2000).

Las autoras feministas pioneras fueron las que plantearon que una intervención basada simplemente en las personas que venden sexo era injusto y discriminatorio (Sullivan, 1992), ya que invisibilizan al cliente y le evadían de responsabilidad, colocando el foco directamente en la persona que vende sexo, recayendo toda la presión del control de los servicios sobre esta. La conducta de vender sexo está estigmatizada mientras que la conducta de comprar lo está mucho menos, encontrando iniciativas siempre centradas en el castigo y rehabilitación de las vendedoras, y secundariamente en la reeducación de quien compra (Juliano, 2002). Escasamente se han realizado intervenciones dirigidas a los hombres que pagan sexo, entre las que podemos distinguir dos tipos de actuaciones.

La primera desde un modelo criminalizador y punitivo, propio de las políticas prohibicionistas como las que se desarrollan en algunos estados de los Estados Unidos, que pretenden acabar con todos los riesgos a través 
de la eliminación de la demanda. Estas acciones de disuasión del consumo se han desarrolladas principalmente en espacios abiertos y visibles (calle), a través de presencia policial en zonas en las que se realizan servicios sexuales para disminuir la presencia de clientes y sancionar el consumo. El desarrollo de las "Escuelas de Johns» han sido una alternativa a la prisión de aquellos hombres que fueron detenidos por solicitar o contratar servicios sexuales. Además, se les ofertan cursos de rehabilitación para evitar la reincidencia en la conducta, o se expone la imagen de estos clientes en webs públicas para que sean estigmatizados, provocando su desprestigio y consecutivamente su disuasión (Monto, 2004). Estas medidas han sido estudiadas y evaluadas y se ha constatado que más que beneficios provocan ocultamiento y desplazamiento de la actividad a otros espacios, sin que se consiga la reducción de la demanda como se esperaba (Campbell y Storr, 2001). En esta línea encontramos que en España se han producido medidas de control y prohibición de la prostitución callejera denominadas Medidas de Convivencia Ciudadanas en los Espacios Públicos (Meneses, 2019). Dictadas por los Ayuntamientos en algunas localidades españolas entre el 2005 al 2015, más exactamente en 51 municipios. Estas medidas no han disuadido la demanda, sino que muchas de las multas recayeron en las propias mujeres, aquellas con mayor vulnerabilidad que eran victimas de trata, con repercusiones muy negativas para las mismas (Villacampa, 2017). Posteriormente, la ley de Seguridad Ciudadana, Ley Orgánica 4/2015 de 30 marzo, conocida como «Ley Mordaza», creo unas condiciones para la persecución tanto de las mujeres que venden servicios sexuales como los hombres que lo compran en todo el Estado, continuando y acrecentando las condiciones de vulnerabilidad de las mujeres. Por otra parte, al centrarse en la prostitución que contacta en la vía pública dejaba fuera el mercado de servicios sexuales de estrato medio y alto, reforzando la jerarquización de la prostitución.

El segundo tipo de intervenciones se sitúa desde la preocupación de disminuir daños, y pretende incidir en aquellos riesgos que se desprenden de la compraventa, tiene su origen en un enfoque educativo sociosanitario, y plantea que las intervenciones no recaigan exclusivamente en las vendedoras sino también en los compradores. Las intervenciones que se han realizado han ido dirigidas a los riesgos mencionados en el punto anterior y que detallamos a continuación.

\section{Medidas sanitarias}

Encontramos actividades que reducen prácticas de riesgo en la salud, mediante intervenciones en clubs, locales y zonas de transito de clientes y 
mediante la oferta de pruebas rápidas de VIH y otras ETS (Diserens et al., 2010). Además, la dispensación de material preventivo, reparto de material informativo, acciones de promoción del uso correcto del preservativo y tratamiento gratuito de ITS dentro de locales han proporcionado resultados muy positivos (Alary et al., 2003). Se ha constatado que el uso de preservativos entre clientes supone una clara disminución de la prevalencia del VIH (Borquez, Hallett, Gomez y Garnett, 2011). Este tipo de intervenciones tienen mejores resultados cuando van de la mano de educación para la salud, ya que la mera distribución de material preventivo podría ser ineficaz (Morio et al., 1999). Por ejemplo, el programa Intervención mejorada en China desarrolló educación de pares, seminarios, servicios de prueba y reuniones sociales que resultaron muy fructíferos, además de dos estudios de vigilancia conductual que consiguieron un mayor uso del condón y una mejora en los comportamientos de riesgo (Lau et al., 2009). Otro ejemplo, es el programa Hombre Seguro en México, a través de intervenciones relacionadas con la reducción de prácticas de riesgo, que pretendía conocer la evolución del consumo de sexo y las prácticas de riesgo asociadas (Pitpitan et al., 2014). Estas intervenciones deben incidir en las relaciones que estos hombres tienen tanto en sus relaciones comerciales como no comerciales (Ompad et al., 2013), especialmente en aquellos contextos en los que haya uso de drogas (Goldenberg et al., 2010), pues benefician a una mayor población (Pitpitan et al., 2014). Los antecedentes de estudio mantienen la necesidad urgente de realizar intervenciones especificas desde el trabajo social con los hombres, ya que puede suponer un gran paso hacía cuestiones como la prevención de enfermedades de transmisión sexual y el VIH (Dizechi et al., 2018), así como su inclusión en la prevención de violencia de género (Karandikar y Gezinski, 2012).

\section{Medidas para reducir la violencia}

Las acciones para mejorar el comportamiento de los clientes, especialmente contra la violencia y el maltrato, han sido llevadas a cabo por organizaciones de trabajadoras sexuales. Encontramos ejemplos a través de Amnesty for Women en Alemania, AMMAR en Argentina o Colectivo Stella en Canadá. A pesar de que son acciones poco desarrolladas desde el trabajo social son positivas porque a los varones se les enseña que sus comportamientos pueden perpetuar diferentes tipos de violencia (Karandikar y Gezinski, 2012). Indudablemente una tarea de concienciación contra la violencia, el respeto y la dignidad hacia las personas que ofertan servicios sexuales está pendiente por desarrollar en profundidad en España. 


\section{Medidas para identificar a víctimas de trata}

Como se ha puesto de relieve en un estudio previo (Meneses et al., 2018b), los hombres que pagan por sexo son los primeros que toman contacto con las víctimas de trata y esta circunstancia debería ser aprovechada para convertirlos en aliados contra la trata. Las acciones dirigidas a la identificación de víctimas de trata con fines de explotación sexual, así como con la identificación de menores en contextos de prostitución, utilizando a los clientes de prostitución, ha sido poco frecuente. Y es que, si bien es cierto que no todos actúan de la misma manera, una gran mayoría de clientes estaría dispuesta a colaborar llamando a la policía (Meneses et al., 2015). Son muchas las experiencias de desarticulación de redes de trata a partir de la denuncia de un cliente. Por lo que la difusión de información sobre indicadores de trata entre los consumidores de sexo de pago, a través de webs de contactos, podría ser una acción educativa a desarrollar. A pesar de ello, está poco explorado el trabajo en el que se haga participe al consumidor en la identificación de víctimas de trata con fines de explotación sexual.

Las intervenciones realizadas en nuestro país son pocas y levemente desarrolladas. Debemos tener en cuenta que el Estado español no tiene una legislación definida en cuanto al fenómeno y el debate sobre la prostitución solo genera posiciones polarizadas que no conducen al fomento de medidas socioeducativas. En relación a la intervención con clientes se han implementado algunas pequeñas actividades dirigidas a reducir prácticas de riesgo, desarrolladas por organizaciones que trabajan con personas que venden sexo y que han considerado los beneficios de ampliar también a los demandantes sus acciones. La Asociación Askabide en Vizcaya, Ámbit Prevenció y Asociación Genera en Barcelona, el Colectivo Hetaira en Madrid, o la Fundación Atenea han sido las protagonistas en esta materia. Como objetivo principal se plantean la búsqueda de mejoras de las condiciones de quienes deben continuar en la actividad y entienden que la intervención con la demanda puede aportar beneficios. Las acciones desarrolladas a veces son solo una ampliación de trabajo que ya se hace y que puede reducirse a sensibilización sobre infecciones de transmisión sexual y de sexo seguro, dispensación de folleos o carteles, anuncios en prensa y en webs y talleres e intervenciones puntuales al demandante. En ocasiones son desarrolladas a través de las propias personas que venden sexo con formación previa y con la difusión de material en locales o a través de la red. 


\section{CONCLUSIONES}

La demanda de servicios sexuales puede producir consecuencias positivas o negativas en las personas que proporcionan estos servicios. Se ha generado principalmente dos tipos de políticas de intervención con los consumidores: la sanción e intento de disuasión del sexo de pago y la intervención socioeducativa y sanitaria directa con los consumidores. Los resultados muestran por el momento como la primera no provoca una mejoría de la realidad en el fenómeno sino todo lo contrario. Puede provocar la dispersión y ocultamiento, aumentando la clandestinidad del fenómeno, y repercutiendo negativamente en las mujeres (Campbell y Storr, 2001). Las organizaciones son conscientes que la sanción directamente a hombres que pagan por sexo no puede más que perjudicar indirectamente a las personas que proporcionan estos servicios (Campbell y Storr, 2001), convirtiendo su situación en más vulnerable. Sin embargo, los resultados obtenidos en la intervención socioeducativa con clientes en relación a prácticas de riesgo han mostrado resultados positivos (Lau et al., 2009). El consumo de sexo de pago es una actividad controlable en la que pueden entrar en juego la educación y la modificación de ciertas actitudes desarrolladas por parte de quienes compran. Además, los perfiles tan variados entre clientes nos muestran que existen hombres más motivados por el contacto personal que establecen con las mujeres y con cierta sensibilidad al bienestar de estas. Los clientes personalizadores, amigos y fijos pueden responder de manera positiva a las intervenciones sociosanitarias.

Se detecta una fuerte carencia de acciones dirigidas a los clientes a nivel general en el contexto español, a pesar de que el trabajo de muchas organizaciones está consolidado, con largas trayectorias y respaldada por administraciones publicas locales. Es necesario mejorar los comportamientos de los varones que pagan por sexo, trabajando desde una perspectiva de género, que genere una sensibilización y concienciación de sus conductas. Deben ser conscientes del peligro que pueden provocar en la salud de las personas que venden sexo, entendiendo la salud desde una perspectiva biopsicosocial y no solo desde el trabajo en la prevención de infecciones de transmisión sexual.

Las intervenciones en los contextos de prostitución y trabajo sexual pueden ir dirigidas en unos casos a la disuasión del consumo de sexo, especialmente entre los menores y hombres más jóvenes, a la reducción de prácticas de riesgo, y a fomentar el buen trato hacía las personas que venden servicios sexuales. Por último, las acciones destinadas a la sensibilización y a la información para la identificación y denuncia de víctimas de trata con fines de explotación sexual son cruciales para la erradicación de la esclavitud sexual. Podemos obtener más beneficios con modelos socioeducativos que punitivos, 
pues solo puede suponer una ampliación de programas ya existentes destinados a la oferta.

\section{REFERENCIAS}

Alario Gavilán, M. (2018). La influencia del imaginario de la pornografía hegemónica en la construcción del deseo sexual masculino prostituyente: un análisis de la demanda de prostitución. ASPARKÍA, 33, 61-79.

Alary, M., Lowndes, C. M., Mukenge-Tshibaka, L., Gnintoungbé, C A B., Bédard, E., Geraldo, N., Jossou, P., Lafia, E., Bernier, F., Baganizi, É., Joly, J. R., Frost, E., y Anagonou, S. (2003). Sexually transmitted infections in male clients of female sex workers in Benin: risk factors and reassessment of the leucocyte esterase dipstick for screening of urethral infections. Sex Transm Infect, 79, 388-392. https://doi. org/10.1136/sti.79.5.388

Askabide, Asociación (2008). Perfil de clientes de prostitución en Bizkaia. Bilbao: Mensajero.

Baumeister, R., y Voh, K. (2004). Sexual Economics: Sex as Female Resource for Social Exchange in Heterosexual Interactions. Personality and Social Psychology Review. 8(4), 339-363.

Belza, M. J., De La Fuente, L., Suárez, M., Vallejo, F., García, M., López, M., Barrio, G., y Bolea, Á. (2008). Men who pay for sex in Spain and condom use: Prevalence and correlates in a representative sample of the general population. Sexually Transmitted Infections, 84(3), 207-211. https://doi.org/10.1136/sti.2008.029827

Berg, R. C., Molin, S., y Nanavati, J. (2020) Women Who Trade Sexual Services from Men: A Systematic Mapping Review, The Journal of Sex Research, 57(1), 104-118, DOI: $10.1080 / 00224499.2019 .1624680$

Borquez, A., Hallett, T. B., Gomez, G. B., y Garnett, G. P. (2011). Condom use by female sex workers and their clients in Mexico: who decides and does it matter? Sexually Transmitted Infections, 87(3), 254-256. https://doi.org/10.1136/ sti.2010.048736

Campbell, R., y Storr, M. (2001). Challenging the kerb crawler rehabilitation programme. Feminist Review, 67(1), 94-108. https://doi.org/10.1080/01417780122701

Centro de Investigaciones Sociológicas (2009). Encuesta Nacional de Salud Sexual.

Church, S., Henderson, M., Barnard, M., y Hart, G. (2000). Violence by Clients Towards Female Prostitutes in Different Work Settings: Questionnaire Survey. British Medical Journal, 2001, 322(3), marzo, 524-525.

Cobo, R. (2016). Un ensayo sociológico sobre la prostitución. Politica y Sociedad, 53(3), 897-914. https://doi.org/10.5209/rev-POSO.2016.v53.n3.48476

Cobo, R. (2017). La prostitución en el corazón del capitalismo. Madrid: Catarata.

Connell, R. W. (2008). A Thousand Miles from Kind: Men, Masculinities and Modern Institutions. The Journal of Men's Studies, 16(3), Fall 2008, 237-252.

Connell, R. W. (2012). Masculinity research and global change. Masculinities and Social Change, 1(1), 4-18. https://doi.org/10.4471/MCS.2012.01 
Cusick, L. (1998). Female Prostitution in Glasgow: Drug Use and Occupational Sector. Addiction Research, 1998. 6(2), 115-30.

De Miguel Álvarez, A. (2014). La prostitución de mujeres, una escuela de desigualdad humana. Dilemata, 6(16), 7-30.

De Miguel Álvarez, A., y Torrado Martín-Palomino, E. (2014). Introducción: Debates y dilemas en torno a la prostitución y la trata. Dilemata (16), 1-6.

Diserens, E.-A., Bodenmann, P., N'Garambe, C., Ansermet-Pagot, A., Vannotti, M., Masserey, E., y Cavassini, M. (2010). Clients of sex workers in Switzerland: it makes sense to counsel and propose rapid test for HIV on the street, a preliminary report. BMC Infectious Diseases, 10(1), 74. https://doi.org/10.1186/14712334-10-74

Dizechi, S., Brody, C., Tuot, S., Chhea, C., Saphonn, V., Yung, K., Kim, S., y Yi, S. (2018). Youth paying for sex: What are the associated factors? Findings from a cross-sectional study in Cambodia. BMC Public Health, 18(1), 1-11. https://doi. org/10.1186/s12889-017-4999-8

Fleming, P. J., Patterson, T. L., Chavarin, C. V, Semple, S. J., Magis-Rodriguez, C., y Pitpitan, E. V. (2017). Behavioral and Psychosocial Correlates of HIV Testing Among Male Clients of Female Sex Workers in Tijuana, Mexico. AIDS and Behavior, 21(8), 2322-2331. https://doi.org/10.1007/s10461-016-1531-6

Giddens, A. (1992). La transformación de la intimidad. Madrid: Cátedra.

Girchenko, P. , Ompad, D. C., Kulchynska, R., Bikmukhametov, D., Dugin, S., y Gensburg, L. (2015). Correlates of Lifetime History of Purchasing Sex Services by Men in Saint Petersburg and Leningrad Oblast, Russia. Journal of Urban Health, 92(6), 1105-1116. https://doi.org/10.1007/s11524-015-9990-z

Goldenberg, S. M., Gallardo Cruz, M., Strathdee, S. A., Nguyen, L., Semple, S. J., y Patterson, T. L. (2010). Correlates of unprotected sex with female sex workers among male clients in Tijuana, Mexico. Sexually Transmitted Diseases, 37(5), 319324. https://doi.org/10.1097/OLQ.0b013e3181c5334f

Gomes Do Espirito Santo, M. E., y Etheredge, G. D. (2005). Male clients of brothel prostitutes as a bridge for HIV infection between high risk and low risk groups of women in Senegal. Sexually Transmitted Infections, 81(4), 342-344. https://doi. org/10.1136/sti.2004.011940

Gómez Suárez, Á., Pérez Freire, S. y ALECRIN, Centro de Día. (2009). Clientes de Prostitución en Galicia: Perfiles y Narrativas Discursivas. Praxis Sociológica. 13, 128-147.

Gómez Suárez, Á., Pérez Freire, S., y Verdugo-Matés, R. M. (2015). El putero español: quienes son y qué buscan los clientes de prostitución en España. Catarata

Gómez Suárez, Á., y Verdugo-Matés, R. M. (2016). Dominación, sexualidad masculina y prostitución en España: ¿por qué los hombres españoles consumen sexo de pago? Convergencia Revista de Ciencias Sociales. 71, 149-174.

Hakim, C. (2010). Erotic Capital. European Sociological Review, 26(5), 499-518.

Hor, L. B., Detels, R., Heng, S., y Mun, P. (2005). The role of sex worker clients in transmission of HIV in Cambodia. International Journal of STD and AIDS, 16(2), 170-174. https://doi.org/10.1258/0956462053057567 
Huysamen, M. (2016). Constructing the 'respectable' client and the 'good' researcher: The complex dynamics of cross-gender interviews with men who pay for sex. Norma, 11(1), 19-33. https://doi.org/10.1080/18902138.2015.1119379

Joseph, L. J., y Black, P. (2012). Who's the Man? Fragile Masculinities, Consumer Masculinities, and the Profiles of Sex Work Clients. Men and Masculinities, 15(5), 486-506. https://doi.org/10.1177/1097184X12458591

Juliano, D. (2002). La prostitución: el espejo oscuro. Barcelona: Icaria Editorial.

Karandikar, S., y Gezinski, L. B. (2012). "Without Us, Sex Workers will Die Like Weeds»: Sex Work and Client Violence in Kamathipura. Indian Journal of Gender Studies, 19(3), 351-371. https://doi.org/10.1177/097152151201900301

Kong, T. S. K. (2015). Romancing the boundary: client masculinities in the Chinese sex industry. Culture, Health and Sexuality. 17(7), 810-824. https://doi.org/10.1080 /13691058.2015.1004197

Lau, J. T. F., Wan, S. P. , Yu, X. N., Cheng, F., Zhang, Y., Wang, N., Zhang, L. y Zhang, J. (2009). Changes in condom use behaviors among clients of female sex workers in China. Sexually Transmitted Infections, 85(5), 376-382. https://doi.org/10.1136/ sti.2008.035162

López Insausti, R., y Baringo Ezquerra, D. (2007). Ciudad y prostitución heterosexual en España: el punto de vista del «cliente» masculino. Documentación Social. 144, 59-74.

Majuelos F., Arjona A., y Checa J. (2019). Una contribución crítica, desde la etnografía, a la construcción categorial del cliente masculino del sexo de pago. Gazeta de Antropología. 35(1), articulo 6.

Majuelos Martínez, F. (2014). Prostitución y sociabilidad. El cliente en perspectiva emic. (Tesis Doctoral). Universidad de Almería, España.

McKeganey, N., y Barnard, M. (1996). Sex Work on The Streets. Prostitutes and Their Clients. Buckingham: Open University Press.

Meneses Falcón, C. (2007a). Consecuencias del uso de cocaína en las personas que ejercen la prostitución. Gac Sanit. 21(3), 191-196.

Meneses Falcón, C. (2007b). Riesgo, vulnerabilidad y prostitución. Documentación Social. 144, 11-35.

Meneses Falcón, C. (2010a). Factores motivacionales en una muestra de hombres españoles que pagan por servicios sexuales. Rev. Asoc. Esp. Neuropsiq, 30(107), 393-407.

Meneses Falcón, C. (2010b). Usos y abusos de drogas en contextos de prostitución. Revista Española de Drogodependencias, 35(3).

Meneses Falcón, C. (2019). ¿Por qué se identifican tan pocas victimas de trata de seres humanos? Revista ICADE. 107, 1-23. doi: 10.14422/icade.i107.y2019.001

Meneses Falcón, C. (2020). ¿La triada adictiva?: consumo de cocaína, alcohol y sexo. Revista Española de Drogodependencias. En prensa.

Meneses Falcón, C., y Rúa-Vieites, A. (2011). Comportamientos de riesgo en los varones que pagan servicios sexuales. Norte de Salud Mental, IX(39), 27-39.

Meneses Falcón C, Rúa-Vieites A, y Uroz-Olivares, J. (2018a). Exploring motives to pay for sexual services from opinions about prostitution. Revista Internacional de Sociología 76(1), e091. https:// doi.org/10.3989/ris.2018.76.2.17.47 
Meneses, C., Uroz, J., y Rua, A. (2018b). Can clients who pay for sexual services help victims of sex trafficking? Masculinities and Social Change, 7(2), 178-208. doi: 10.17583/MCS.2018.3173

Meneses Falcón, C., Uroz Olivares, J., Rúa Vieites, A., Gortázar Rotaeche, C., y Castaño Reyero, M. J. (2015). Apoyando a las víctimas de trata. Delegación del Gobierno para la Violencia de Género. Ministerio de Sanidad, Servicios Sociales e Igualdad.

Millett, K. (2018). Política Sexual. Colección feminismos. Madrid, Ediciones Catedra

Monto, M. (2004). Female Prostitution, Customers, and Violence. Violence Against Women. 10(2), 160-188.

Moreira, Isabel Cristina Cavalcante Carvalho y Monteiro, Claudete Ferreira de Souza. (2012). La violencia en el cotidiano de la prostitución: invisibilidades y ambigüedades. Revista Latino-Americana de Enfermagem 20(5), 954-960.

Morio, S., Soda, K., Tajima, K., Leng, H. B., Kitamura, K., Mizushima, S., Ohshige, Tan, F., Suyama, A., Sopheab, H., y Phalla, T. (1999). Sexual Behaviour of Commercial Sex Workers and Their Clients in Cambodia. Journal of Epidemiology. 9(3), 175-182. Retrieved from https://www.jstage.jst.go.jp/article/jea1991/9/3/9_3_175/_ pdf/-char/en

Mulieri, I., Santí, F., Colucci, A., Fanales-Belasio, E., Gallo, P., y Luzi, A. M. (2010). Sex workers clients in Italy: results of a phone survey on hiv risk behaviour and perception. Ann Ist Super Sanità, 50(4), 363-368. https://doi.org/10.4415/ANN

Neira, M. (2012). Una mala mujer: la prostitución al descubierto. Barcelona: Plataforma.

Ompad, D. C., Bell, D. L., Amesty, S., Nyitray, A. G., Papenfuss, M., Lazcano-Ponce, E., Villa, Luisa L., y Giuliano, A. R. (2013). Men Who Purchase Sex, Who Are They? An Interurban Comparison. Journal of Urban Health: Bulletin of the New York Academy of Medicine, 90(6), 1166-1180. https://doi.org/10.1007/s11524-0139809-8

Pardo Herrero, E., Meroño Salvador, M., y Fundació Àmbit Prevenció. (2015). Estudio Cualitativo Sobre Clientes De La Prostitución. Barcelona.

Pitpitan, E. V., Chavarin, C. V, Semple, S. J., Magis-Rodriguez, C., Strathdee, S. A., y Patterson, T. L. (2014). Hombre Seguro (Safe Men): A sexual risk reduction intervention for male clients of female sex workers. BMC Public Health, 14(1), 1-12. https://doi.org/10.1186/1471-2458-14-475

Raphael, J., y Shapiro, D. (2004). Violence in Indoor and Outdoor Prostitution Venues. Violence Against Women, 2004. 10(2), 126-39.

Sanders, T. (2004). A continuum of risk? The management of health, physical and emotional risks by female sex workers. Sociology of Health \& Illness. 26(5), 557574.

Sanders, T. (2008). Paying for pleasure. Men who buy sex. Cullompton, Devon: Willan Publishing.

Simonetto, P. (2018). Pagar para ser hombre. Prácticas y sentidos de la compra de sexo en los testimonios judiciales de trabajadores. Provincia de Buenos Aires, 1936-1960. Revista Historia y Justicia. 10, 14-41. https://doi.org/10.4000/rhj.1275

Solana, J. L. (2003). La prostitución, tráfico e inmigración de mujeres. Granada: Ed. Comares. 
Sullivan, J. (1992). Local government and community in Java: An urban case study. USA: Oxford University Press.

Villacampa, C. (2017). Municipal ordinances and street prostitution in Spain. Eur. J. Crim. Policy Res., 23, 41-57.

Wahab, S., \& Sloan, L. (2000). Feminist voices on sex work: Implications for social work. Affilia, 15(4), 457-479.

Weitzer R. (2012). Sex Trafficking and the Sex Industry: The Need for. EvidenceBased Theory and Legislation. The journal of criminal law and criminology. 101(4), 1337-1370.

Yang, Y., Yang, C., Latkin, C., Luan, R., y Nelson, K. E. (2016). Condom Use during Commercial Sex among Male Clients of Female Sex Workers in Sichuan China: A social cognitive theory analysis HHS Public Access. AIDS Behav, 20(10), 23092317. https://doi.org/10.1007/s10461-015-1239-z 